\title{
SCENARIO OF CERVICAL PAIN IN YOUNG IT PROFESSIONALS DUE TO SPONDYLOTIC CHANGES, PREVENTION, CONTROL AND MANAGEMENT
}

\author{
Gourab Maji \\ PT. BPT(WBUHS), CMT \\ Sr. Consultant, Department of Physiotherapy, \\ South Dum Dum Municipal Hospital, Kolkata, India
}

\begin{abstract}
There was increasing number of cases about neck pain in last few years at IT places. The reason behind the pain was prolonged neck flexing which leads to early Cervical Spondylosis, nerve root compression and radiculopathy to arms. Young IT professionals were affected major by this unavoidable condition due to lot of pressure, stress and postural dysfunctions. These mechanical changes can cause severe damage in future. Young generations should be cautious and aware about the symptom and nature of pain, which might be cured by early preventions, control measures and physiotherapy management.
\end{abstract}

Keywords: Neck Pain, Cervical Spondylosis, Physiotherapy management.

\section{INTRODUCTION}

Neck pain is very common in young professionals specially in IT sectors. They are engaged for 7-8 hours in front of computer server with prolonged neck flexion. They cannot avoid the work pressure and nature of postural habit. In the beginning 1-2 years of their job, they might not feel any dysfunction or pain. But after 4-5 years they feel the arising of the pain and stiffness around the neck joint. Sometimes the pain radiates at upper back, paraspinal area, shoulder and arm regions. The average case study is seen around age limit between 28 to 40 years of age. The clinical presentation and $\mathrm{x}$-ray diagnosis are described below in a table, that how severity of the pain increases with the age of work in IT industry.

\begin{tabular}{|l|l|l|}
\hline Age Limit & Clinical Presentation & X-Ray Diagnosis \\
\hline 28-32 yrs. & $\begin{array}{l}\text { - Pain mild to moderate. } \\
\text { - Minimum radiation. } \\
\text { - Muscular stiffness. } \\
\text { - Muscle spasm. }\end{array}$ & $\begin{array}{l}\text { - Minimum loss of curvature in } \mathrm{C}_{\mathrm{x}} \text { spine. } \\
\text { - Mild reduce intervertebral space at } \mathrm{C}_{4}-\mathrm{C}_{7} \\
\text { level. }\end{array}$ \\
\hline 32-35 yrs. & $\begin{array}{l}\text { - Pain moderate to severe. } \\
\text { - Moderate radiation to arms. } \\
\text { - Muscular stiffness. } \\
\text { 35-40 yrs. }\end{array}$ & $\begin{array}{l}\text { - Muscle spasm. } \\
\text { - Pain severe. } \\
\text { - Severe radiculopathy in arm, forearm, loss of curvature in } \mathrm{C}_{\mathrm{x}} \text { spine. } \\
\text { fingers, numbness. } \\
\text { - Vertigo, Muscle spasm. } \\
\text { - Muscular stiffness. }\end{array}$ \\
\hline
\end{tabular}

Cervical Spondylosis: Cervical $\left(\mathrm{C}_{\mathrm{x}}\right)$ Spine consist of 7 vertebrae $C_{1}$ to $C_{7}$. The space between two vertebrae is called intervertebral space. The curvature of $\mathrm{C}_{\mathrm{x}}$ spine is called lordotic curvature. Each intervertebral space contain an intervertebral disc. All the nerves of upper limb are arised from 
nerve roots of this spinal column. Prolonged neck flexion creates a long time biomechanical force at curvature of $C_{x}$ spine. Gradually it leads to straightening of normal curvature. This condition arises with neck muscle stiffness, spasm and pain. The loss of curvature leads to decrease the intervertebral space. The reduction of space creates a pressure from forward to backward direction on intervertebral disc. In severe Cervical Spondylosis, the intervertebral disc is pressurized to bulge at posterior and postiro-lateral direction of $\mathrm{C}_{\mathrm{x}}$ spine. The posterior direction of dise bulge cause narrowing of spinal canal is called Spinal Canal Stenosis. Whether the postero-lateral dise bulge creates a pressure on nerve roots of $\mathrm{C}_{\mathrm{x}}$ spine, which leads to Cervical radiculopathy. Vertigo is often seen in severe Spondylotic changes, due to compression on arterial supply on brain.

\section{METHOD}

Clinical intervention is very important to prevent the progress of the condition. This method is classified in 3 major steps assessment, diagnosis and treatment procedures.

- The motor and sensory function should be assessed by proper protocol. The motor function is tested by passive and active movement of neck and shoulder. If pain arises in particular movement like neck flexion, extension or lateral rotation, it should be noted. Range of motion should be marked in all directions. Sensory function is assessed by fine touch and deep pressure on dermatome. Pain on palpations or numbness may present. Past history of any trauma or direct injury on affected area should be noted.

- Diagnostic tests are referred to confirm the exact locations of root cause. X-ray can show the loss of curvature or intervertebral space reductions or any abnormal pathology of bone. MRI is useful more to detect the locations of nerve root compression, the measurement of intervertebral space reductions, spinal canal stenosis or prolapsed intervertebral disc (PIVD). Electrodiagnosis test like Electromyography (EMG) or Nerve Conduction Velocity (NCV) test is required to detect the affected nerve and muscle in radiculopathy.

- Treatment or intervention can be classified in prevention, control and physiotherapy management.

- Prevention is very necessary step to control the progress of symptom. Prolonged neck flexion should be avoided more than 30 mins. Regular exercise for neck and shoulder joint are useful method. Thin pillow should be used during sleep.
- The pain can be controlled by exercises and preventive measures. Proper postural habit might reduce pain. Avoid any jerk or further injury on neck joint.

- $\quad$ Physiotherapy Management - Physiotherapy is a better conservative method to relief from this pain. Proper postural advice is prescribed in ergonomics training at IT Sectors. The young professionals are guided to use desktop computer or laptop in proper way. Static neck exercises are advised to reduce the pain, radiculopathy and vertigo. Dynamic strengthening exercises are helpful to improve the muscle strength around the neck and shoulder. Stretching of muscles group like flexors, extensors and lateral rotators of neck and shoulder is useful method to decrease the muscle stiffness and spasm. Electrotherapy intervention is important aspect of physiotherapy management. Ultrasound therapy, laser therapy and TENS are useful method to reduce pain, stiffness and radiculopathy. Manual therapy is also a modern aspect of intervention.

\section{RESULTS}

The outcome of physiotherapy intervention and postural care is familiar and satisfactory. The early Spondylosis is cured completely by proper following of guidelines. Modulate to severe neck pain is also reduced by right postural habit, exercises and review. $70-80 \%$ of young IT professionals get positive result in 10-15 sittings of physiotherapy in a clinic or hospital. They are advised to continue the home exercises program to control the condition.

\section{CONCLUSION}

To conclude the overall scenario it is undoubtedly accepted that the prognosis of Cervical pain and radiculopathy is very common at IT sectors, specially in young professionals. It affects the efficiency of work and psychological condition. Physiotherapy intervention play a significant role to prevent, control and manage the pathology. Due to rapid prosperity of IT sector more young peoples will be employed gradually in future. As a result it is important to notify the upcoming stress of work. It is better to understand the involvement of Cervical spine in IT work. A physiotherapist can guide a conservative method to prevent the severity of Cervical Spondylosis. This will help to prevent surgical intervention in future.

\section{REFERRENCES}


International Journal of Engineering Applied Sciences and Technology, 2021

Vol. 6, Issue 1, ISSN No. 2455-2143, Pages 240-242

Published Online May 2021 in IJEAST (http://www.ijeast.com)

1.Susan B. O'Sullivan, PT, EdD;Thomas J.

Schmitz, PT, PhD;Physical Rehabilitation, nerve root pain, 952

2.S.Brent Brotzman, MD; Robert C. Manske, PT, DPT,SCS,MEd, ATC, CSCS; Clinical Orthopaedic

Rehabilitation:an evidenced based approach;Christopher J.Durall, PT, DPT, MS, SCS,

LAT, CSCS:Therapeutic exercise for cervical spine. 